\title{
A Systematic Approach to Overcome the Matrix Effect during LC-ESI-MS/ MS Analysis by different Sample Extraction Techniques
}

\author{
Chinmoy Ghosh ${ }^{1,2 *}$, Shashank Gaur ${ }^{1}$, Chandrakant P. Shinde ${ }^{2}$ and Bhaswat Chakraborty ${ }^{1}$
}

${ }^{1}$ Bioanalytical Department, Research and Development, Cadila Pharmaceuticals Limited, 1389-Trasad Road, Dholka, Gujarat, India

${ }^{2}$ School of Studies in Chemistry, Jiwaji University, Gwalior, M.P., India

\begin{abstract}
Matrix effect (ME) is a major concern during any LC-ESI-MS/MS analysis. It affects reproducibility, sensitivity and reliability of these analytical techniques. There are, however, no standardized approaches available to overcome the ME related issues. The approach varies from molecule to molecule. Here we examined that sample extraction technique is an approach to overcome the ME during LC-MS/MS bioanalysis. Three types of conventional extraction techniques i.e. protein precipitation, liquid-liquid extraction and solid phase extraction (SPE) were used during extraction of nevirapin from human plasma. It was observed that nevirapin sample when prepared by SPE, eliminates or reduces dramatically the MEs ipso facto. Protein precipitated samples showed highest degree of ME with average matrix factor of 0.30 ; liquid-liquid extraction had an average matrix factor of 0.80 ; and the matrix factor obtained by solid phase extraction was 0.99 . Different phospholipids were identified by performing the precursor ion scanning at $\mathrm{m} / \mathrm{z} 104$ and 184, which interfered with the analyte during chromatographic elution. From the experiment it was observed that long retained phospholipids had significant role on ME. So it is a good practice to observe the phospholipids during precursor scanning for a period which is at least three times of the actual analytical run time. Among all the applied extraction techniques, solid phase extraction produces the cleanest sample, and methanol precipitation produces the dirtiest sample due to high solubility of the phospholipids into it.
\end{abstract}

Keywords: Matrix effect; Extraction technique; Protein precipitation; Liquid-liquid extraction; Solid phase extraction; LC-ESI-MS/MS; Nevirapin; Plasma samples

\section{Introduction}

The term "matrix effect" (ME) is in vogue these days in the context of LC-MS/MS bioanalytical methods. It is defined as the suppressive or enhancing effect of any component other than the molecule of interest at its retention. ME is fast becoming a very important topic of discussion among the bioanalysts all over the world. Even the regulatory authorities are emphasizing on MEs during their inspection of Bioanalytical laboratories. In the third and fourth regulated Crystal City bioanalysis conferences [1,2], MEs and incurred sample analysis (ISR) were the major topics of discussion.

Recent advances in drug discovery, clinical research and formulation development have enabled researchers to develop more potent and efficacious molecules resulting in reduction of dosage strength from milligrams to micrograms. As such, it is essential to develop more sensitive and reliable bioanalytical methods, which can provide the actual pharmacokinetic profile of the newly formulated molecule. MEs may play a very important role to achieve this required sensitivity. If the intended analytical method has MEs in terms of ion suppression, it will be very difficult to achieve the desired sensitivity as the low signals will be suppressed even beyond the average detector noise. On the other hand, presence of ion enhancement in the analytical method may lead to misleading lower limit of quantitation (LLOQ) and lower concentration values for the intended method.

During every LC-MS/MS bioanalysis it is essential to estimate, minimize or eliminate the MEs. MEs are determined in terms of matrix factor (MF) which is widely known. MF is the ratio of the responses obtained from the analyte spiked into post extracted blank matrix to the analyte spiked into the clean solution. MF of more than unity $(>1)$ indicates the ion enhancement, $\mathrm{MF}<1$ indicates the ion suppression, whereas $M F=1$, means the analytical method is free from any MEs. If the analytical method exhibits any ME, then it is recommended to eliminate or minimize it as much as possible. Regarding causality of MEs, there are a few that have been reported till date. Among them, the prominent causes are as follows: firstly, chromatographic conditions $[8,12,29]$, which shows gradient method, selective column and mobile phase with less additives is better option to overcome the MEs [317]. Secondly, applied ionization technique, as it was reported that ESI is more prone to MEs than the APCI [4,5,10,12,14,18-21]. This is mainly because of the different ion formation mechanism in both the techniques. Thirdly, ionization polarity, since positive polarity showed more MEs in comparison with negative polarity [14,22]; and perhaps the most important source of $\mathrm{ME}$ is the inefficient extraction technique used to extract the analyte and/or metabolite from the specified matrix [3-7,10-12,14-18,23-30]. Ion suppression or enhancement is not the only indications of MEs, but retention time shifts due to deposition of phospholipids inside the analytical column, elevated baseline because of the presence of different phospholipids in the extracted samples, fluctuating calibration curves [29,31], and inconsistent responses due to inconsistent ionization, are also caused due to the presence of ME in the applied analytical method.

This manuscript describes a systematic approach towards elimination of the MEs by using the suitable extraction techniques. The approaches are explained in details with graphical illustrations at

*Corresponding author: Chinmoy Ghosh, Research Scientist, Research and Development, Cadila Pharmaceutical Limited, 1389, Trasad Road Dholka-387 810, Ahmedabad, Gujarat, India, E-mail: chinmoy_ghosh@ yahoo.com,drb.chakraborty@cadilapharma.co.in

Received April 23, 2011; Accepted July 21, 2011; Published July 23, 2011

Citation: Ghosh C, Gaur S, Shinde CP, Chakraborty B (2011) A Systematic Approach to Overcome the Matrix Effect during LC-ESI-MS/MS Analysis by different Sample Extraction Techniques. J Bioequiv Availab 3: 122-127. doi:10.4172/jbb.1000072

Copyright: (C) 2011 Ghosh C, et al. This is an open-access article distributed under the terms of the Creative Commons Attribution License, which permits unrestricted use, distribution, and reproduction in any medium, provided the original author and source are credited. 
each step. During the study of MEs, different phospholipids were also studied which were observed during different extraction procedures.

\section{Experiment}

\section{Apparatus and software}

The HPLC system with an auto sampler was a Shimadzu SILHTc (Shimadzu, Japan) and solvent delivery module was LC-10ADvp coupled with Applied Biosystem (MDS Sciex, ON, Canada) API 3000 Tandem mass spectrometer as the detection system. Electrospray ionization source (ESI) was attached with the MS/MS system. All chromatographic integrations were performed by the Analyst software (version: 1.4.2; Applied Biosystems, ON, Canada). Hand vortexer used for protein precipitation was procured from Remi (Mumbai, India). Multi-pulse vortexer used for liquid-liquid extraction was purchased from Glas-Col (Cole Parmer, USA). The Caliper turbovap LV concentration workstation that was used to evaporate the samples was purchased from Caliper Life Sciences (Hopkinton, MA, USA). Positive pressure unit used for SPE was obtained from Orochem technologies Inc (Lombard, IL, USA).

\section{Chemicals and reagents}

Nevirapin (NVP) was procured from Cadila Pharmaceutical Ltd., Dholka, Ahmedabad, India. Formic acid, ethyl acetate and methyl tertiary butyl ether (MTBE) were procured from Merck Specialities Pvt. Ltd, Mumbai, India. Water used was collected from water purification systems (Milli Q, Milli Pore, USA) installed in our laboratory. Methanol and acetonitrile were of HPLC grade and were supplied by J. T. Baker, USA. Fresh frozen human plasma ( $\mathrm{K}_{2}$-EDTA as anticoagulant) was used during sample analysis, and was supplied by Clinical department of Cadila Pharmaceuticals Limited, Dholka, Ahmedabad, India. Plasma was stored at $-70 \pm 5^{\circ} \mathrm{C}$. Oasis HLB $30 \mathrm{mg}, 1 \mathrm{cc}$ cartridge was procured from Waters Corporation India (Ahmedabad, India) and Lichrosep, $30 \mathrm{mg}, 1 \mathrm{~mL}$ cartridges were purchased from Merck India Limited (Mumbai, India).

\section{Standards and working solutions}

Individual stock standard solution of NVP, containing $1 \mathrm{mg} / \mathrm{mL}$, was prepared by dissolving pure compound in methanol. The intermediate and working solutions of NVP were prepared from corresponding stock solutions by diluting with a mixture of water: methanol 30:70 $\mathrm{v} / \mathrm{v}$ as a diluent. Samples of two different concentration levels, i.e. low concentration level [LCL] $(30.00 \mathrm{ng} / \mathrm{mL})$, and high concentration level [HCL] $(3900.40 \mathrm{ng} / \mathrm{mL})$, were also prepared. All these stock solutions and different concentration samples were stored at $4 \pm 2^{\circ} \mathrm{C}$.

\section{Chromatographic conditions}

Chromatographic separation was performed on a Hypurity advance C18, 50 x $4.6 \mathrm{~mm} \mathrm{ID,} 5 \mu$ particle size, analytical column (Thermo Electron Corporation, U.K.) and the mobile phase was a mixture of $0.10 \%(\mathrm{v} / \mathrm{v})$ formic acid in water to acetonitrile at a ratio of $15: 85, \mathrm{v} / \mathrm{v}$. Injection volume was $5 \mu \mathrm{L}$. The flow rate was $0.500 \mathrm{~mL} / \mathrm{min}$. Total analysis time of a single injection was 3.00 minutes. Temperatures of column oven and auto sampler were set at $40^{\circ} \mathrm{C}$ and $5^{\circ} \mathrm{C}$, respectively.

\section{Mass spectrometric conditions}

Detection was carried out using a triple quadrupole tandem mass spectrometer (API 3000, MDS Sciex, ON, Canada) equipped with electrospray interface (ESI). Ions were created in positive ion mode setting the sprayer voltage at $4.0 \mathrm{kV}$ and the ion source temperature at $400^{\circ} \mathrm{C}$. The typical ion source parameters, viz., declustering potential, collision energy, entrance potential, focusing potential and collision cell exit potential were 50,39,15, 400 and $16 \mathrm{~V}$. Nitrogen gas was used as gas 1 , gas 2 , curtain gas and collision-activated dissociation gas, which were set at 12, 8, 6 and $12 \mathrm{l} / \mathrm{min}$, respectively. Detection of the ions was performed in the multiple reaction monitoring (MRM) modes, monitoring the NVP ion with transition: $\mathrm{m} / \mathrm{z} 267.0 \rightarrow 226.2$ with a dwell time of $300 \mathrm{msec}$. As mentioned above, the analytical data were processed by Analyst software (Version 1.4.2; Applied Biosystems).

\section{Extracted samples for studying ME}

Different extraction techniques were used to extract NVP from human plasma. Extraction was started with protein precipitation using methanol and acetonitrile separately. In this method $10 \mu \mathrm{L}$ of NVP (or $10 \mu \mathrm{L}$ of diluents in case of blank sample) standard was added to $190 \mu \mathrm{L}$ of plasma blank. Then $600 \mu \mathrm{L}$ of methanol or $400 \mu \mathrm{L}$ of acetonitrile were added into it, the sample was vortexed for 1 minute followed by centrifugation at $15000 \mathrm{rpm}$ for 5 minutes at $5^{\circ} \mathrm{C}$. The supernatant was collected and $5 \mu \mathrm{L}$ injected directly into LC-MS/MS.

In liquid-liquid extraction procedure, $10 \mu \mathrm{L}$ of NVP (or $10 \mu \mathrm{L}$ of diluents in case of blank sample) standard was added to $190 \mu \mathrm{L}$ of plasma blank, and extracted with ethyl acetate and MTBE as extracting solvent. $3 \mathrm{~mL}$ of this extracting solvent was added to each sample during extraction. Then the samples were vortexed in multipulse vortexer for 5 minutes, followed by centrifugation at $5000 \mathrm{rpm}$ for 5 minutes at $5^{\circ} \mathrm{C}$. The organic supernatant was collected and dried under the stream of nitrogen at $40^{\circ} \mathrm{C}$. The dried samples were reconstituted with $200 \mu \mathrm{L}$ of mobile phase and $5 \mu \mathrm{L}$ injected into LC-MS/MS.

The third extraction technique for studying ME was a solid phase extraction method which was used to extract the NVP from human plasma samples. $10 \mu \mathrm{L}$ of NVP sample was added to $190 \mu \mathrm{L}$ of plasma blank and the sample was vortexed for 15 seconds, SPE cartridge was conditioned with $1 \mathrm{~mL}$ of methanol, followed by equilibration with $1 \mathrm{~mL}$ of Milli-Q water. Then the sample was loaded and washed two times with $1 \mathrm{~mL}$ of Milli-Q water. This step was followed by drying the cartridge under nitrogen for $1 \mathrm{~min}$. and the sample was eluted with $1 \mathrm{~mL}$ of mobile phase and injected directly into LC-MS/MS system.

\section{Results and Discussion}

MEs were studied separately in all three extraction techniques mentioned above. In each case, ME was measured qualitatively and quantitatively. Qualitative experiment was performed by post column infusion technique. In this technique aqueous solution of NVP was infused directly into the MS/MS system along with an injection of extracted plasma blank sample through the LC. The sharp fall of the response at the retention time of NVP, confirms the presence of MEs i.e. ion suppression. These MEs are, at least in major part, due to the presence of different phospholipids in the extracted plasma samples. This is why further investigation was initiated to find out those phospholipids which were responsible for causing MEs.

\section{Monitoring phospholipids}

Glycerophosphocholines and lysophosphophatidylcholines represent respectively the $70 \%$ and $10 \%$ of the total plasma phospholipids [10] and are the major source that can cause MEs. For this reason, seven naturally occurring phospholipids, including two lysophospholipids, were selected to monitor phospholipids removal from biological extracts. Most phospholipids have common product ions at $\mathrm{m} / \mathrm{z} 104$ and 184 . The MRM transitions used for determining the presence of the major phospholipids in the extracted plasma samples were: $\mathrm{m} / \mathrm{z} \quad 496 \rightarrow 184 \& 524 \rightarrow 184$ for lysophosphophatidylcholines, 
and $\mathrm{m} / \mathrm{z} \quad 704 \rightarrow 184,758 \rightarrow 184,786 \rightarrow 184,806 \rightarrow 184 \& 823.4 \rightarrow 184$ for glycerophosphocolines. Along this MRM transitions, one more MRM transition was added into it i.e. $\mathrm{m} / \mathrm{z} 184 \rightarrow 184$, to check whether $\mathrm{m} / \mathrm{z}$ 184 precursor ions get generated in the ion source (Figure 1(included as supplementary data)). The general principles of phospholipids fragmentation were described by Brugger et al. [32], who demonstrated that a positive-ion mode precursor-ion scan of $\mathrm{m} / \mathrm{z} 184$ is specific for phosphocholine-containing phospholipids, i.e., phosphatidylcholine and sphingomyelin. Thelysophosphatidylcholine product-ion spectrum in the positive-ion mode displayed several ions originating from the collision-induced dissociation of the phosphocholine head group, which includes the most intense peak at $\mathrm{m} / \mathrm{z} 184$ and comparatively a less intense peak at $\mathrm{m} / \mathrm{z} 104$. Since lysophosphatidylcholine represents only $10 \%$ of the total plasma phospholipids so, m/z 104 was not included into MRM scan transition.

The MRM transitions as mentioned in the preceding paragraph will detect only lysophosphophatidylcholines (lyso$\mathrm{PC})$, glycerophosphocolines (PC) and sphingomyelin (SM). This technique cannot be used to monitor the other phospholipids, such as phosphotidylethanilamine (PE), phosphotidylinositol (PI), phosphotidylglycerol (PG), phosphotidylserine (PS) and phosphatidic acid (PA), since these phospholipids do not generate the $\mathrm{m} / \mathrm{z} 184$ ion in the source. Consequently, to identify any phospholipids other than the above mentioned MRM transitions, precursor ion scan at m/z 104 and $\mathrm{m} / \mathrm{z} 184$ was performed in positive polarity in ESI interface. For every extracted blank sample, precursor ion scanning was continued for 10 minutes to observe the presence of any long retained phospholipids even after the elution of NVP from the column.

\section{Quantitation of matrix effect}

For quantitative determination of ME, MF was calculated. MF was calculated at low and high concentration level samples. To prepare these matrix factor samples, the plasma blank samples were prepared as per the above mentioned extraction procedures in the respective extraction techniques. From six different plasma lots, four plasma blank samples were processed from each lot. Then $10 \mu \mathrm{L}$ of NVP solution of low concentration and high concentration levels were spiked into $200 \mu \mathrm{L}$ of extracted plasma blank. Two samples at each level from each plasma lot were prepared by using those four extracted plasma blanks respectively. So, total 12 post extracted low concentration level samples and 12 post extracted high concentration level samples were prepared for the study of MF. Similarly, $10 \mu \mathrm{L}$ of NVP solution at low and high concentration levels were spiked into $200 \mu \mathrm{L}$ of mobile phase to prepare the un-extracted sample.

All the experiments presented in this manuscript were categorized into three different cases. Case 1, demonstrates all data related to protein precipitation; Case 2, describes about liquid-liquid extraction and the finally, Case 3, talks about the data related to solid phase extraction technique.

\section{Case -1 (Protein precipitation)}

Protein precipitation was conducted by using two different precipitating agents, i.e., methanol and acetonitrile.

Study of matrix effects in methanol precipitated samples: Initially NVP was attempted to be extracted from human plasma by protein precipitation with methanol as precipitating agent. After protein precipitation and centrifugation, the supernatant was directly injected into LC-MS/MS system. The observed chromatographic responses at the low concentration level of extracted samples were low showing poor chromatographic peaks. Figure 2(included as supplementary data) shows the chromatogram of methanol precipitated samples at low concentration level. Since the response was very low, the presence of ion suppression was suspected. Further, to check the MEs, post column infusion experiment was performed. The post column infusion chromatogram showed sharp decrease of the responses at the retention time of NVP (Figure 3 (included as supplementary data), which qualitatively confirms the presence of MEs i.e. ion suppression. For quantitative estimation of ME, matrix factor was calculated from post extracted spiked samples at low and high concentration levels. The obtained MFs at low concentration level and high concentration level samples were 0.16 and 0.43 , respectively. The average MF of all samples was 0.30 and the overall CV of measurements was $46 \%$. These data confirm the presence of MEs i.e. ion suppression in methanol precipitated extraction techniques. So, further investigation was carried out to find the phospholipids causing this ME.

As a part of the investigation process, precursor ion scan at m/z 184 was monitored, to observe different phospholipids upto 10 minutes of an analytical run. From the obtained spectra, the precursor ions were extracted at the retention window of NVP ( $\sim 1-2$ mins.), as well as, from the complete analytical run. In the retention window of the analyte, phospholipids with $\mathrm{m} / \mathrm{z}$ 497.4, 523.4, 759.9, 785.6 and 811.7 were observed, whereas, in the total analytical run time window, phospholipids at $\mathrm{m} / \mathrm{z} 497.5,521.4,759.8,787.5$ and 811.7 were present (Figure 4(included as supplementary data)). Again, precursor ion scan at $\mathrm{m} / \mathrm{z} 104$ was also performed to observe if any other phospholipids were present. Though no phospholipids were found at the retention window of analyte, but after the elution of NVP, many phospholipids were eluted from the column. Those were $\mathrm{m} / \mathrm{z} 497.6,521.5,523.5$, 525.7, 543.6, 545.5, 548.0 and 567.3. Phospholipids observed in region C, D, and E in Figure 5, had very high intensities.

Methanol precipitated plasma blank, when analyzed by using the above mentioned 8 pairs of MRM transition method, three PCs at $\mathrm{m} / \mathrm{z}$ 704, 758 and 786 showed sharp falls in the responses at the retention window of NVP.

Study of matrix effects in acetonitrile precipitated samples: Having observed very high degree of suppression of NVP molecular ion while using methanol as precipitating agent, it was extracted from human plasma using acetonitrile as precipitating agent. Thereafter, ME was studied by post column infusion, which also showed sharp decrease of the responses at the retention time of NVP, like methanol extracted samples.

This observation qualitatively confirms the presence of MEs i.e. ion suppression. Thus, before further investigation was started to identify those phospholipids, causing the ion suppression, matrix factor was calculated from post extracted spiked samples at low and high concentration levels for quantitative confirmation of MEs. The obtained MF at low concentration level and high concentration level was 0.16 and 0.36 respectively. The average MF was 0.26 with overall \% CV was 41.47. These data confirm the presence of MEs i.e. ion suppression in this extraction technique.

Investigation into ion suppression began with precursor ion scan at $\mathrm{m} / \mathrm{z} 184$, during 10 minutes of analytical span. From the obtained spectra, parent ions were extracted at the retention window of NVP $(\sim$ 1-2 mins.), as well as, from complete analytical cycle. In the retention window of analyte, phospholipids with $\mathrm{m} / \mathrm{z} 759.7,760.8,783.6,785.6$, 787.6 and 811.8 were observed, whereas, in the total analytical run window, phospholipids at $\mathrm{m} / \mathrm{z} 759.8,785.6$ and 811.7 were present 
(Table 1). Again, precursor ion scan at $\mathrm{m} / \mathrm{z} 104$ was also performed to observe if any other phospholipids were present. Though no phospholipids were found at the retention window of analyte, but after the analyte elution, many phospholipids were eluted from the column. Those were $\mathrm{m} / \mathrm{z} 497.8,521.6,523.8,525.7,543.6,545.6,547.6,564.0$, and 567.6 (Table 1). Moreover, when the precipitated plasma blank was analyzed using the above mentioned 8 pairs of MRM transition method, two PCs at $\mathrm{m} / \mathrm{z} 758$ and 786 showed sharp fall in the responses at the retention window of NVP, whereas the other phospholipids did not show any major changes in the response.

\section{Case -2(Liquid-liquid extraction)}

Like protein precipitation, liquid-liquid extraction was also performed in two different extraction solvents i.e. ethyl acetate and MTBE.

Study of matrix effects in liquid-liquid extracted samples by ethyl acetate: NVP was extracted from human plasma by liquidliquid extraction technique using ethyl acetate as extracting solvent. Post column infusion of extracted plasma blank sample was carried out to visualize the presence of any MEs. The obtained chromatogram showed decrease in the responses at the retention time of NVP (Figure 6(included as supplementary data)), but comparatively lesser than that of protein precipitated samples. Followed by post column infusion MF was calculated. The observed MF of post extracted-spiked samples which were extracted using ethyl acetate was 0.75 for low concentration level samples and 0.84 for HCL samples. The average matrix factor was calculated as 0.79 (which includes 12 low concentration level and 12 high concentration level samples) with \% CV of 6.68.

Since, ion suppression was observed in the post column infusion chromatography, so, the investigation process was started with precursor ion scan at $\mathrm{m} / \mathrm{z} 184$, during 10 minutes of analytical span. From the obtained spectra, parent ions were extracted at the retention window of NVP ( 1-2 mins.), as well as, from complete 10 minutes of analysis time. In the retention window of analyte, phospholipids with $\mathrm{m} / \mathrm{z}$ 759.7, 783.6, 785.6, 788.2, 811.6 and 815.2 were observed, whereas, in the total analytical run window, many other phospholipids of $\mathrm{m} / \mathrm{z}$ 497.4, 521.3, 523.4, 787.6, 790.5 and 811.8 were present (Table 1). Again, precursor ion scan at $\mathrm{m} / \mathrm{z} 104$ was also performed to observe if any more phospholipids were present. Though no phospholipids with high intensities were found at the retention window of analyte, but after the analyte elution, many phospholipids were eluted from the column. Those phospholipids were $\mathrm{m} / \mathrm{z} 497.6,521.6,523.5,525.6,543.7,545.7$, 547.6, and 568.3 (Table 1).

Moreover, when the liquid-liquid extracted plasma blank was analyzed using the above mentioned 8 pairs of MRM transition method, two PCs at m/z 758 and 786 showed slight fall of the responses at the retention window of NVP.

Study of matrix effects in liquid-liquid extracted samples by MTBE: MTBE was used as liquid-liquid extraction solvent to extract NVP from human plasma. After the extraction, ME was checked through the post column infusion mode. The observed chromatogram and the calculated MF were almost similar to that of results obtained by ethyl acetate extraction. The observed chromatographic responses at the low concentration level extracted samples were good enough with good chromatographic peak (Figure 7 (included as supplementary data)). The observed MF of low concentration level sample was 0.76 and 0.83 for HCL samples. The average matrix factor was calculated as 0.80 (which includes 12 low concentration level and 12 high concentration level samples) with \% CV of 6.14 .
Hence, as a part of investigation process, precursor ion scan at $\mathrm{m} / \mathrm{z}$ 184 was monitored, to observe different phospholipids upto 10 minutes of analytical run. From the obtained spectra, parent ions were extracted at the retention window of NVP ( 1-2 mins.), as well as, from complete analytical run. During the analytical run phospholipids with $\mathrm{m} / \mathrm{z} 759.8$, $760.6,786.3,787.5$, and 811.7 were observed. Again, precursor ion scan at $\mathrm{m} / \mathrm{z} 104$ was also performed to observe if any other phospholipids were present. No phospholipids with good intensities were present during the whole analytical run (Table 1).

Moreover, when the extracted plasma blank was analyzed using the above mentioned 8 pairs of MRM transition method; two PCs at $\mathrm{m} / \mathrm{z} 758$ and 786 showed slightly downward responses at the retention window of NVP.

\section{Case $\mathbf{- 3}$ (Solid phase extraction)}

Since both the above mentioned techniques have MEs related problems, and the objective of this manuscript is to show the effect of different extraction techniques on MEs, so NVP was extracted from human plasma by solid phase extraction technique. In our laboratory we used two different make SPE cartridges. One was Oasis HLB $30 \mathrm{mg}$, $1 \mathrm{cc}$ cartridge procured from Waters Corporation India (Ahmedabad, India) and the other one was Lichrosep Sequence $30 \mathrm{mg}, 1 \mathrm{~mL}$ cartridge from Merck India Ltd. (Mumbai, India). The extraction technique was same for both the cartridges as mentioned in the section 2.6.

Study of matrix effects of SPE samples (Lichrosep sequence, $30 \mathrm{mg}, 1 \mathrm{~mL}$ ): During the study of MEs in solid phase extraction technique, the calculated MF was 0.98 and 0.97 at low concentration level and high concentration level. The average MF was 0.98 with $\% \mathrm{CV}$ of 1.44 . The measured value was also confirmed by the observed chromatogram obtained by post column infusion, which showed no sign of MEs (Figure 8(included as supplementary data)). The observed phospholipids during the precursor ion scan at $\mathrm{m} / \mathrm{z} 184$ were: $\mathrm{m} / \mathrm{z}$ 759.8, 786.5, 787.5, and 811.8 (Table 1). Whereas during precursor ion scan at $\mathrm{m} / \mathrm{z} 104$ the obtained phospholipids were of $\mathrm{m} / \mathrm{z} 497.8$, 521.8, 543.3, and 545.5 (Table 1). The observed phospholipids were very less in comparison with other extraction techniques mentioned above. The chromatogram of solid phase extracted low concentration level sample showed very good chromatography (Figure 9(included as supplementary data)).

Moreover, when the extracted plasma blank was analyzed using the above mentioned 8 pairs of MRM transition method none of the phospholipids were responsible for ion suppression (Figure 10(included as supplementary data)).

Study of matrix effects of SPE samples (Oasis HLB $30 \mathrm{mg}, 1 \mathrm{cc}$ ): When ME was studied using Oasis HLB cartridges, no major differences were observed in comparison with lichrosep cartridges. The calculated MF was 0.99 for both low concentration level and high concentration level samples. The average MF was also 0.99 with $\% \mathrm{CV}$ of 1.92 . The measured value was also confirmed by the observed chromatogram obtained by post column infusion, which showed no ion suppression, even the chromatography obtained from extracted LCL samples were also good (Figure 11(included as supplementary data)). The observed phospholipids during the precursor ion scan at $\mathrm{m} / \mathrm{z} 184$ were: $\mathrm{m} / \mathrm{z} 759.8$, $783.5,785.8,787.5$, and 811.6 (Table 1). Whereas, during precursor ion scan at $\mathrm{m} / \mathrm{z} 104$, the obtained phospholipids were of $\mathrm{m} / \mathrm{z} 497.5,521.6$, 523.6, and 543.9 (Table 1). The observed phospholipids were very less in comparison with other extraction techniques mentioned above. 


\begin{tabular}{|c|c|c|c|c|c|c|}
\hline \multirow{4}{*}{$\begin{array}{l}\text { Different extraction } \\
\text { techniques }\end{array}$} & \multicolumn{5}{|c|}{ Observed phospholipids } & \multirow{4}{*}{$\begin{array}{c}\text { MF } \\
(\% \mathrm{CV})^{*}\end{array}$} \\
\hline & \multicolumn{2}{|c|}{ Retention window of nevirapin } & \multicolumn{2}{|c|}{ Complete analysis time } & \multirow[t]{2}{*}{$\begin{array}{c}\text { Known phospholipids } \\
\text { responsible for } \mathrm{ME}^{*}\end{array}$} & \\
\hline & \multicolumn{4}{|c|}{ Pre-cursor ion scanning at } & & \\
\hline & $\mathrm{m} / \mathrm{z} 104$ & $\mathrm{~m} / \mathrm{z} 184$ & $\mathrm{~m} / \mathrm{z} 104$ & $\mathrm{~m} / \mathrm{z} 184$ & $\mathrm{~m} / \mathrm{z} 184$ & \\
\hline Methanol precipitation & Nil & $\begin{array}{c}497.4,523.4,759.9 \\
785.6 \text { and } 811.7\end{array}$ & $\begin{array}{c}\text { 497.6, 521.5, 523.5, 525.7, } \\
543.6,545.5,548.0 \text { and 567.3 }\end{array}$ & $\begin{array}{c}497.5,521.4,759.8 \\
787.5 \text { and } 811.7\end{array}$ & $\begin{array}{c}704(\mathrm{PC}) \\
758(\mathrm{PC}) \text { and } 786(\mathrm{PC}) .\end{array}$ & $0.30(\% \mathrm{CV} 46.0)$ \\
\hline Acetonitrile precipitation & Nil & $\begin{array}{c}\text { 759.7, } 760.8,783.6 \\
785.6,787.6 \text { and } 811.8\end{array}$ & $\begin{array}{c}497.8,521.6,523.8,525.7 \\
543.6,545.6,547.6,564.0 \\
\text { and } 567.6\end{array}$ & $59.8,785.6$ and 811.7 & $758(P C)$ and $786(P C)$. & 0.26 (\%CV 41.47) \\
\hline $\begin{array}{l}\text { Liquid-liquid extraction with } \\
\text { ethyl acetate }\end{array}$ & Nil & $\begin{array}{c}\text { 759.7, 783.6, 785.6, } \\
788.2,811.6 \text { and } 815.2\end{array}$ & $\begin{array}{c}497.6,521.6,523.5,525.6 \\
543.7,545.7,547.6 \text {, and 568.3. }\end{array}$ & $\begin{array}{c}\text { 497.4, 521.3, 523.4, } \\
787.6,790.5 \text { and } 811.8\end{array}$ & $758(P C)$ and $786(P C)$ & $0.79(\% \mathrm{CV} 6.68)$ \\
\hline $\begin{array}{c}\text { Liquid-liquid extraction with } \\
\text { methyl tertiary butyl ether }\end{array}$ & Nil & Nil & Nil & $\begin{array}{c}759.8,760.6,786.3 \\
787.5, \text { and } 811.7\end{array}$ & $758(P C)$ and $786(P C)$. & $0.80(\% \mathrm{CV} 6.14)$ \\
\hline $\begin{array}{c}\text { Solid phase extraction } \\
\text { (Merck, Lichrosep catridge) }\end{array}$ & Nil & $759.9,787.7$ and 811.7 & $497.8,521.8,543.3$, and 545.5 & $759.8,787.5$, and 811.8 & Nil & $0.98(\% \mathrm{CV} 1.44)$ \\
\hline $\begin{array}{c}\text { Solid phase extraction } \\
\text { (Waters, Oasis HLB } \\
\text { catridge) }\end{array}$ & Nil & $\begin{array}{c}759.7,783.5,787.5 \text { and } \\
811.7\end{array}$ & $497.5,521.6,523.6$, and 543.9 & $759.8,785.8$ and 811.6 & Nil & $0.99(\% \mathrm{CV} 1.92)$ \\
\hline
\end{tabular}

\#: Complete analysis time excludes the retention window of nevirapin.

$\neq:$ Phospholipids showed ion suppression during the MRM scan of these 7 pairs at $\mathrm{m} / \mathrm{z}: 496 \rightarrow 184,524 \rightarrow 184,704 \rightarrow 184,758 \rightarrow 184,786 \rightarrow 184,806 \rightarrow 184 \& 823.4 \rightarrow 184$

$¥$ : Overall CV obtained from 12 low concentration level samples and 12 high concentration level samples.

Table 1: Observed phospholipids during different extraction techniques.

Moreover, when the extracted plasma blank was analyzed using the above mentioned 8 pairs of MRM transition method; none of the phospholipids showed any sign of ion suppression (Figure 12(included as supplementary data)).

\section{Discussion}

In order to systematically examine MEs on NVP, the latter was extracted from human plasma using different extraction techniques. Specifically the role and extent of interfering influence of phospholipids were studied. During protein precipitation, it was observed that more phospholipids were extracted by methanol than acetonitrile. This is because most of the phospholipids were more soluble in methanol than acetonitrile. When precursor ions scan at m/z 104 of first plasma blank sample was performed upto its actual analytical run time of 3 minutes, no phospholipids were observed, but from the subsequent injection, phospholipids at the retention window of NVP was present. Consequently, precursor ion scan was performed upto 10 minutes to check any long runner, which revealed the late elution of some phospholipids from the column. The reason behind this late elution from the column is that phosphatidylcholine containing phospholipids consist of both polar head group. This polar head contains a negatively charged phosphate group and a positively charged quaternary amine group, and one or two long alkyl chain(s). The polar head group impart strong ionic character to the phospholipids, while the long alkyl chains make them extremely hydrophobic, often requiring a hold at nearly $100 \%$ organic solvent to elute them from a reversed-phase chromatographic column [10].

The late elution time may not present a problem for polar analytes, as they are not expected to co-elute with the phospholipids. Nonpolar analytes, however, run a significant risk of co-elution with these phospholipids. In addition, residual phospholipids, if not eluted from the analytical column, can build up on the column and significantly reduce the column longevity. So, in this situation either the run time should be adjusted in such a way so that retained phospholipids do not co-elute with the analyte or some gradient method can be used to remove those phospholipids in every run from the column.

Since protein precipitation showed high ion suppression, so NVP was extracted by liquid-liquid extraction. More polar ethyl acetate, extracts more phospholipids compare to less polar MTBE, though the MEs were similar in both the extracting solvents. From the precursor ion scanning, it was also observed that there was no late eluting phospholipid for MTBE as extracting solvent. Whereas, some late eluting phospholipids were observed in case of samples extracted with ethyl acetate. So, less polar solvents produce cleaner extracted samples. Since samples extracted with liquid-liquid extraction technique presented ME, NVP samples were further extracted by solid phase extraction technique. There was no ME in the samples extracted by SPE, the cleanest samples were obtained by this technique. Both the cartridges produced the same results, so it is recommended to use Lichrosep Sequence $30 \mathrm{mg}, 1 \mathrm{~mL}$ cartridges, since it is more cost effective in comparison with Oasis HLB $30 \mathrm{mg}, 1 \mathrm{cc}$ cartridges. From all extracted samples it was observed that methanol extracts the maximum phospholipids and Oasis HLB cartridge extracts the least amount of phospholipids (Figure 13(included as supplementary data)).

\section{Conclusion}

Late eluting phospholipids may have detrimental effects on recovery, sensitivity, reproducibility and the most important reliability of the analytical data. So it recommended that during method development, while checking the presence of any phospholipids, set aside the required time to study ME thoroughly for the sake of enhanced reliability of the method. Moreover, among conventional extraction techniques, solid phase extraction technique appears to be the best option in most of the cases, as exemplified by nevirapin in this study [33]. It prepares the cleanest samples and avoids ME. 


\section{Acknowledgement}

Authors would like to thank Mrs. Koyel Ghosh, for her valuable time to correct and modify the language of the manuscript. Authors also like to thank all bioanalytical staff for their support to this research work.

\section{References}

1. Guidance for Industry and FDA (2007) Staff - Assayed and Unassayed Quality Control Material, Food and Drug Administration, CDRH 01-13.

2. Savoie N, Garofolo F, Van Amsterdam P, Bansal S, Beaver C, et al. (2010) White Paper on Recent Issues in Regulated Bioanalysis \& Global Harmonization of Bioanalytical Guidance. Bioanalysis 2: 1945-1960

3. Taylor PJ (2005) Matrix effects: The Achilles heel of quantitative high-performance liquid chromatography-electrospray-tandem mass spectrometry. Clin Biochem 38: 328-334.

4. Smeraglia J, Baldrey SF, Watson D (2002) Matrix Effects and Selectivity Issues in LC-MS-MS. Chromatographia 55: S95-S99.

5. Annesley TM (2003) Ion Suppression in Mass Spectrometry. Clin Chem 49: 1041-1044.

6. Fu I, Woolf EJ, Matuszewski BK (1998) Effect of the Sample Matrix on the Determination of Indinavir in Human Urine by HPLC with Turbo Ion Spray Tandem Mass Spectrometric Detection. J Pharmaceut Biomed Anal 18: 347 357.

7. Jemal M, Ouyang Z, Yuan-Qing X (2010) Systematic LC-MS/MS Bioanalytical method development that incorporates plasma phospholipids risk avoidance, usage of incurred sample and well thought-out chromatography. Biomed Chromatogr 24: 2-19.

8. Du L, White RL (2008) Reducing glycerophosphocholine lipid matrix interference effects in biological fluid assays by using high-turbulance liquid chromatography. Rapid Commun Mass Sppectrom 22: 3362-3370.

9. Steene JCVD, Lambert WE (2008) Comparison of Matrix Effects in HPLCMS/MS and UPLC-MS/MS Analysis of Nine Basic Pharmaceuticals in Surface Waters. J Am Soc Mass Spectrom 19: 713-718.

10. Chambers E, Wagrowski-Diehl DM, Lu Z, Mazzeo JR (2007) Syetematic and comprehensive strategy for reducing matrix effects in LC/MS/MS analysis. J Chromatogr B Analyt Technol Biomed Life Sci 852: 22-34.

11. Annesley TM (2007) Methanol-Associated Matrix Effects in Electrospray Ionization Tandem Mass Spectrometry. Clin Chem 53: 1827-1834.

12. Gosetti F, Mazzucco E, Zampieri D, Gennaro MC (2010) Signalsuppression/ enhancement in high-performance liquid chromatography tandem mass spectrometry. J Chromatogr A 1217: 3929-3937.

13. Omnia I, Halquist MS, Elmamly MY, Shalaby A, Kames HT (2007) Monitoring phospholipids for assessment of matrix effects in a liquid chromatographytandem mass spectrometry method for hydrocodon and pseudoephedrine in human plasma. J Chromatogr B Analyt Technol Biomed Life Sci 859: 84-93.

14. Ismaiel OA, Halquist MS, Elmamly MY, Shalaby A, Thomas Karnes $H$ (2008) Monitoring phospholipids for assessment of ion enhancement and ion suppression in ESI and APCI LC/MS/MS for chlorpheniramine in human plasma and the importance of multiple source matrix effect evaluations. $J$ Chromatogr B Analyt Technol Biomed Life Sci 875: 333-343.

15. Mei H, Hsieh Y, Nardo C, Xu X, Wang S, et al. (2003) Investigation of Matrix Effects in Bioanalytical High-Performance Liquid Chromatography/Tandem Mass Spectrometric Assays: Application to Drug Discovery. Rapid Commun Mass Spectrom 17: 97-103.

16. Mallet CR, Ziling L, Mazzeo JR (2004) A study of ion suppression effects in electrospray ionization from mobile phase additives and solid-phase extracts. Rapid Commun Mass Spectrom 18: 49-58.

17. Matuszewski BK, Constanzer ML, Chavez-Eng CM (2003) A Systematic Approach to Reducing Matrix Effects in LC/MS/MS Analyses. Anal Chem 75: 3019-3030

18. Dams R, Huestis MA, Lambert WE, Murphy CM (2003) Matrix Effect in BioAnalysis of Illicit Drugs with LC-MS/MS: Influence of Ionization Type, Sample Preparation, and Biofluid. J Am Soc Mass Spectrom 14: 1290-1294.

19. Mei H, Korfmacher W (Ed.) (2005) Using Mass Spectrometry for Drug Metabolism Studies. CRC Press, Boca Raton, FL, 103-150.
20. Liang HR, Foltz RL, Meng M, Bennett P (2003) lonization enhancement in atmospheric pressure chemical ionization and suppression in electrospray ionization between target drugs and stable-isotope-labeled internal standards in quantitative liquid chromatography/tandem mass spectrometry. Rapid Commun. Mass Spectrom 17: 2815-2821

21. Souverain S, Rudaz S, Veuthey JL (2004) Matrix effect in LC-ESI-MS and LC-APCI-MS with off-line and on-line extraction procedures. J Chromatog A 1058: 61-66.

22. Ghosh C, Shinde CP, Chakraborty BS (2010) Ionization Polarity as a Cause of Matrix Effects, its Removal and Estimation in ESI-LC-MS/ MS Bio-analysis J Anal Bioanal Techniques 1: 106.

23. Buhrman DL, Price PI, Rudewicz PJ (1996) Quantitation of SR27417 in Human Plasma Using Electrospray Liquid Chromatography Tandem Mass Spectrometry: A Study of Ion Suppression. J Am Soc Mass Spectrom 7 1099-1105.

24. Pucci V, Palma SD, Alfieri A, Bonelli F, Monteagudo E (2009) A novel strategy for reducing phospholipids-based matrix effect in LC-ESI-MS bioanalysis by means of Hybrid SPE. J. Pharmaceut. Biomed Anal 50: 867-871.

25. Shena JX, Motykab RJ, Roachb JP, Hayesa RN (2005) Minimization of ion suppression in LC-MS/MS analysis through the application of strong cation exchange solid-phase extraction (SCX-SPE). J Pharmaceut Biomed Anal 37 359-367.

26. Chin C, Zhang ZP, Karnes HT (2004) A study of matrix effects on an LC/MS/ MS assay for olanzapine and desmethyl olanzapine. J Pharmaceut Biomed Anal 35: 1149-1167.

27. Avery M (2003) Quantitative characterization of differential ion suppression on liquid chromatography/atmospheric pressure ionization mass spectrometric bioanalytical methods. Rapid Commun Mass Spectrom 17: 197-201.

28. Marchese A, McHugh C, Kehler J, Bi H (1998) Determination of Pranlukast and its metabolites in human plasma by LC/MS/MS with PROSPEKT on-line solid-phase extraction. J Mass Spectrom 33: 1071-1079.

29. Bennett P, Hairui (2004) Overcoming Matrix Effects Resulting From Biological Phospholipids Through Selective Extraction in Quantitative LC/ MS/MS. Presented at the ASMS Conference.

30. Bonfiglio R, King R, Olah T, Merkle K (1999) The effects of sample preparation methods on variability of the electrospray ionization response for model drug compounds. Rapid Commun Mass Spectrom 13: 1175-1185.

31. Capka V, Carter SJ (2006) Non-intuitive Strategies for Minimizing Matrix Effects in Development of Ultrasensitive Bioanalytical LC/MS/MS Methods. Presented at the ASMS Conference.

32. Brugger B, Erben G, Sandhoff R, Wieland FT, Lehmann WD (1997) Quantitative analysis of biological membrane lipids at the low picomole leve by nano-electrospray ionization tandem mass spectrometry. Proc Natl Acad Sci USA 94: 2339-2344.

33. Ghosh C, Gaur S, Singh A, Shinde CP, Chakraborty BS (2011) Estimation of Nevirapine from Human Plasma by LC-ESI-MS/MS: a Pharmacokinetic Application. J Bioequiv Availab 3: 020-025. 